BARI-TH/97-250

May 1997

\title{
QCD Interactions of Heavy Mesons with Pions by Light-Cone Sum Rules
}

\author{
P. Colangelo ${ }^{1}$ and F. De Fazio ${ }^{1,2}$ \\ ${ }^{1}$ I.N.F.N., Sezione di Bari, Italy \\ ${ }^{2}$ Dipartimento di Fisica, Universitá di Bari, Italy
}

\begin{abstract}
Light-cone QCD sum rules are employed to compute the strong coupling constants: $g_{B^{*} B^{*} \pi}, g_{B_{1} B_{0} \pi}$ and $g_{B_{1} B^{*} \pi}$, where $\left(B, B^{*}\right)$ and $\left(B_{0}, B_{1}\right)$ are negative and positive parity $\left(0^{-}, 1^{-}\right)$and $\left(0^{+}, 1^{+}\right) \bar{q} Q$ doublets. The couplings are calculated both for finite values of the heavy quark mass and in the infinite heavy quark mass limit, deriving sum rules for $m_{Q} \rightarrow \infty$.
\end{abstract}

PACS:11.55.Hx,13.20.He 


\section{INTRODUCTION}

The recognition of the approximate symmetries of QCD, explicitely or spontaneously broken, represents a basic step for the understanding and the description of the strong interactions in processes involving hadrons. A remarkable example, in the sector of systems containing one heavy quark, is the $S U\left(2 N_{f}\right)$ heavy flavour-spin symmetry holding in the infinite heavy quark mass limit: $m_{Q_{f}} \rightarrow \infty$. In this limit, the masses of the $N_{f}$ heavy flavoured quarks are irrelevant in the interactions with the hadronic light degrees of freedom, since the heavy quarks only act as static colour sources. Moreover, the decoupling of the gluon from the spin $s_{Q}$ of the heavy quark allows to relate the properties of the states belonging to the doublets obtained combining $s_{Q}$ with the spin $s_{\ell}^{P}$ of the light degrees of freedom. The $S U\left(2 N_{f}\right)$ spin-flavour symmetry is explicitely broken by the finite values of the heavy quark masses, and the symmetry-breaking effects can be described using an expansion in the inverse masses of the heavy quarks [1,2].

At the opposite energy scale, for vanishing masses of the up, down and strange quarks, QCD is invariant under chiral $S U(3)_{L} \times S U(3)_{R}$ transformations; this symmetry is spontaneously broken, the Goldstone bosons being represented by the octet of light pseudoscalar mesons [3].

Both the heavy quark spin-flavour and the chiral symmetries can be realized writing an effective QCD lagrangian, expressed in terms of hadronic fields 四. The interactions of the heavy $s_{\ell}^{P}=\frac{1}{2}^{-}$and $\frac{1}{2}^{+}$mesons with the octet of light pseudoscalar $0^{-}$mesons can be described by the lagrangian:

$$
\begin{aligned}
\mathcal{L} & =i \operatorname{Tr}\left\{H_{b} v^{\mu} D_{\mu b a} \bar{H}_{a}\right\}+\frac{f_{\pi}^{2}}{8} \operatorname{Tr}\left\{\partial^{\mu} \Sigma \partial_{\mu} \Sigma^{\dagger}\right\}+\operatorname{Tr}\left\{S_{b}\left(i v^{\mu} D_{\mu b a}-\delta_{b a} \Delta\right) \bar{S}_{a}\right\} \\
& +i g \operatorname{Tr}\left\{H_{b} \gamma_{\mu} \gamma_{5} \mathcal{A}_{b a}^{\mu} \bar{H}_{a}\right\}+i g^{\prime} \operatorname{Tr}\left\{S_{b} \gamma_{\mu} \gamma_{5} \mathcal{A}_{b a}^{\mu} \bar{S}_{a}\right\}+\left[i h \operatorname{Tr}\left\{S_{b} \gamma_{\mu} \gamma_{5} \mathcal{A}_{b a}^{\mu} \bar{H}_{a}\right\}+\text { h.c. }\right] .
\end{aligned}
$$

The fields $H_{a}$ in (1.1) describe the negative parity $J^{P}=\left(0^{-}, 1^{-}\right) \bar{q} Q$ doublets (with $s_{\ell}^{P}=\frac{1}{2}^{-}$):

$$
H_{a}=\frac{\left(1+y^{\prime}\right)}{2}\left[P_{a \mu}^{*} \gamma^{\mu}-P_{a} \gamma_{5}\right]
$$


where the operators $P_{a}^{* \mu}$ and $P_{a}$ respectively annihilate the $1^{-}\left(B_{a}^{*}\right)$ and $0^{-}\left(B_{a}\right)$ mesons of four-velocity $v(a=u, d, s$ is a light flavour index). In the infinite heavy quark mass limit such states are degenerate in mass, due to the vanishing of the chromomagnetic interaction of the heavy quark spin with the spin of the light degrees of freedom of the mesons. Hence, $H_{a}$ describes a doublet of states with the light degrees of freedom with zero orbital angular momentum with respect to the heavy quark. Analogously [5], the fields $S_{a}$ describe a doublet of states having the light degrees of freedom with angular momentum $s_{\ell}=\frac{1}{2}^{+}(P$-waves in the constituent quark model):

$$
S_{a}=\frac{1+\Varangle}{2}\left[D_{1}^{\mu} \gamma_{\mu} \gamma_{5}-D_{0}\right]
$$

with $D_{1}^{\mu}, D_{0}$ annihilation operators for the states $1^{+}$and $0^{+}$, respectively. Notice that all the heavy field operators in the lagrangian (1.1) contain a factor $\sqrt{m_{P}}$ and have dimension $3 / 2$; the parameter $\Delta$ in (1.1) represents the mass splitting between the positive and negative parity states.

The octet of light pseudoscalar mesons is included in the effective lagrangian (1.1) using the exponential representation: $\xi=e^{\frac{i \mathcal{M}}{f_{\pi}}}$ and $\Sigma=\xi^{2}$; the matrix $\mathcal{M}$ contains the $\pi, K$ and $\eta$ fields:

$$
\mathcal{M}=\left(\begin{array}{ccc}
\sqrt{\frac{1}{2}} \pi^{0}+\sqrt{\frac{1}{6}} \eta & \pi^{+} & K^{+} \\
\pi^{-} & -\sqrt{\frac{1}{2}} \pi^{0}+\sqrt{\frac{1}{6}} \eta & K^{0} \\
K^{-} & \bar{K}^{0} & -\sqrt{\frac{2}{3}} \eta
\end{array}\right)
$$

with $f_{\pi}=132 \mathrm{MeV}$. Finally, the operators $D$ and $\mathcal{A}$ in (1.1) are given by:

$$
\begin{aligned}
& D_{\mu b a}=\delta_{b a} \partial_{\mu}+\mathcal{V}_{\mu b a}=\delta_{b a} \partial_{\mu}+\frac{1}{2}\left(\xi^{\dagger} \partial_{\mu} \xi+\xi \partial_{\mu} \xi^{\dagger}\right)_{b a} \\
& \mathcal{A}_{\mu b a}=\frac{1}{2}\left(\xi^{\dagger} \partial_{\mu} \xi-\xi \partial_{\mu} \xi^{\dagger}\right)_{b a} .
\end{aligned}
$$

The effective Lagrangian (1.1) can be generalized to include the interactions of heavy $s_{\ell}^{P}=\frac{3}{2}^{+}$states, as well as the interaction with the octet of low-lying vector mesons [6].

The strong interactions between heavy $H_{a}, S_{a}$ mesons and the light pseudoscalar mesons, as described by (1.1), are determined by three couplings: $g, h$ and $g^{\prime}$. The spin symmetry 
implies that the couplings to light mesons (from now on we refer to pions) do not depend on the particular member of a doublet; for example, the couplings $g_{B^{*} B^{*} \pi}$ and $g_{B^{*} B \pi}$, defined respectively by the matrix elements

$$
<\bar{B}^{* 0}\left(p, \epsilon_{2}\right) \pi^{-}(q) \mid B^{*-}\left(p+q, \epsilon_{1}\right)>=\frac{2 i}{f_{\pi}} g_{B^{*} B^{*} \pi} \epsilon_{\rho \sigma \alpha \beta} \epsilon_{1}^{\rho} \epsilon_{2}^{* \sigma} p^{\alpha} q^{\beta}
$$

and

$$
<\bar{B}^{0}(p) \pi^{-}(q) \mid B^{*-}(p+q, \epsilon)>=\frac{2 m_{B}}{f_{\pi}} g_{B^{*} B \pi} \epsilon^{\mu} q_{\mu}
$$

( $\epsilon$ is the $B^{*}$ polarization vector), coincide with the same coupling $g$ in the limit $m_{b} \rightarrow \infty$. Analogously, the constant $h$ describes the coupling to pions of whatever a member of the $H_{a}$ doublet and a member of the $S_{a}$ one, whereas the strong interaction of positive parity $S_{a}$ states with pions is governed by the coupling $g^{\prime}$.

Differences between the couplings of states belonging to the same doublet are of order $1 / m_{Q}$. Such differences play an important role in the heavy meson phenomenology; for example, the difference between the pion-vector-vector $g_{B^{*} B^{*} \pi}$ and the pion-pseudoscalar-vector $g_{B^{*} B \pi}$ coupling constant appears in the $1 / m_{Q}$ correcting terms to the effective lagrangian (1.1) [7], and must be taken into account in the calculation of the heavy meson hyperfine splitting [8,9].

In refs. 10 12 the couplings $g$ and $h$ were computed using QCD sum rules and the shortdistance expansion [13, in the infinite heavy quark mass limit. The short-distance expansion was also adopted to calculate the couplings $g_{B^{*} B \pi}, g_{B^{*} B^{*} \pi}$ and $g_{B_{0} B \pi}$ for finite masses of the heavy quarks 114,10,11,9, 15]; comparing the results derived in the asymptotic regime and for finite masses, significant information was obtained on the size of $1 / m_{Q}$ corrections.

A different approach to compute the strong couplings was proposed in [16] and adopted in [11,17], using sum rules based on the expansion near the light-cone [18 20] The parameters

\footnotetext{
1 Applications of the light-cone sum rule method to semileptonic heavy meson decays can be found in [21] and in references therein.
} 
$g_{B^{*} B \pi}, g_{B_{0} B \pi}$ were determined by this technique for finite masses of the charm and bottom quarks, and the couplings $g$ and $h$ were obtained extrapolating to $m_{Q} \rightarrow \infty$ the results for finite $m_{c}$ and $m_{b}$.

In this paper we derive light-cone QCD sum rules in the limit $m_{Q} \rightarrow \infty$. This allows us to perform a systematic comparison between the results of light-cone and short-distance QCD sum rules, which can shed light on the critical parameters, the advantages and the drawbacks of both the approaches. As we shall see, in the infinite heavy quark mass limit a remarkable agreement between the two approaches is obtained, supporting our confidence on such methods for the calculation of parameters which are sensitive to the nonperturbative dynamics of QCD.

The plan of the paper is as follows. In Sec. II we apply light-cone sum rules to calculate the coupling $g$ and $g_{B^{*} B^{*} \pi}$; the numerical analysis of this channel is presented in Sec. III, together with a comparison with the results obtained for $g_{B^{*} B \pi}$. In Sec. IV and Sec. V we analyze the couplings $g^{\prime}$ and $h$, respectively; in particular, we consider the process $B_{1} \rightarrow B_{0} \pi$. In Sec. VI we draw our conclusions.

\section{LIGHT-CONE QCD SUM RULES FOR $g_{B^{*} B^{*} \pi}$ AND $g$}

Let us first consider the finite heavy mass case, and compute the coupling $g_{B^{*} B^{*} \pi}$ defined in eq.(1.7). The correlator of two vector currents $J_{\mu}=\bar{q} \gamma_{\mu} b$ between the vacuum state and a pion external state with momentum $q$ :

$$
\begin{aligned}
F_{\mu \nu}(p, q) & =i \int d x<\pi^{-}(q)\left|T\left[\bar{d}(x) \gamma_{\mu} b(x), \bar{b}(0) \gamma_{\nu} u(0)\right]\right| 0>e^{i p x} \\
& =i \epsilon_{\mu \nu \alpha \beta} p^{\alpha} q^{\beta} F
\end{aligned}
$$

corresponds to the diagram depicted in Fig.1. Considering a pion on mass shell: $q^{2}=m_{\pi}^{2}$ (we set $m_{\pi}=0$ ), the invariant function $F$ depends on $p^{2}$ and $(p+q)^{2}$.

Following the same idea underlying the usual QCD sum rule method, a hadronic representation can be given to the correlator (2.1) in terms of the lowest lying resonance, the $B^{*}$ 
pole, of higher resonances and a continuum of states. In a double dispersion relation for $F$ in the variables $p^{2}$ and $(p+q)^{2}$ :

$$
F\left(p^{2},(p+q)^{2}\right)=\int d s d s^{\prime} \frac{\rho^{h a d}\left(s, s^{\prime}\right)}{\left(s-p^{2}\right)\left(s^{\prime}-(p+q)^{2}\right)},
$$

the spectral function $\rho^{\text {had }}$ gets contributions from $B^{*}$ and from higher states; therefore, it can be modeled as

$$
\rho^{\text {had }}\left(s, s^{\prime}\right)=\frac{2}{f_{\pi}} g_{B^{*} B^{*} \pi} m_{B^{*}}^{2} f_{B^{*}}^{2} \delta\left(s-m_{B^{*}}^{2}\right) \delta\left(s^{\prime}-m_{B^{*}}^{2}\right)+\rho^{\text {cont }}\left(s, s^{\prime}\right) \theta\left(s-s_{0}\right) \theta\left(s^{\prime}-s_{0}^{\prime}\right)
$$

where $\rho^{\text {cont }}\left(s, s^{\prime}\right)$ includes the contribution of the higher states and of the continuum above the effective thresholds $s_{0}, s_{0}^{\prime}$; the $B^{*}$ leptonic constant $f_{B^{*}}$ in (2.3) is defined as

$$
<0\left|\bar{d} \gamma_{\mu} b\right| \bar{B}^{* 0}(p, \epsilon)>=m_{B^{*}} f_{B^{*}} \epsilon_{\mu} .
$$

We neglect in the dispersion relation (2.2) possible subtraction terms, which play no role in the Borel transformed sum rules, as shown in the following.

For large positive values of $-p^{2}$ and $-(p+q)^{2}$ the function $F\left(p^{2},(p+q)^{2}\right)$ can be computed in QCD. This task is afforded expanding the T-product in eq.(2.1) near the light-cone $\left(x^{2}=0\right)$ in terms of non local operators, whose matrix elements are defined by wave functions associated with operators of increasing twist. According to the notations in ref. [16], the catalog of the relevant matrix elements, up to twist 4 , is:

$$
\begin{aligned}
<\pi(q)\left|\bar{d}(x) \gamma_{\mu} \gamma_{5} u(0)\right| 0> & =-i f_{\pi} q_{\mu} \int_{0}^{1} d u e^{i u q x}\left(\varphi_{\pi}(u)+x^{2} g_{1}(u)+\mathcal{O}\left(x^{4}\right)\right) \\
& +f_{\pi}\left(x_{\mu}-\frac{x^{2} q_{\mu}}{q x}\right) \int_{0}^{1} d u e^{i u q x} g_{2}(u), \\
<\pi(q)\left|\bar{d}(x) i \gamma_{5} u(0)\right| 0> & =\frac{f_{\pi} m_{\pi}^{2}}{m_{u}+m_{d}} \int_{0}^{1} d u e^{i u q x} \varphi_{P}(u), \\
<\pi(q)\left|\bar{d}(x) \sigma_{\mu \nu} \gamma_{5} u(0)\right| 0> & =i\left(q_{\mu} x_{\nu}-q_{\nu} x_{\mu}\right) \frac{f_{\pi} m_{\pi}^{2}}{6\left(m_{u}+m_{d}\right)} \int_{0}^{1} d u e^{i u q x} \varphi_{\sigma}(u) .
\end{aligned}
$$

The wave function $\varphi_{\pi}$ is associated with the leading twist 2 operator, $g_{1}$ and $g_{2}$ correspond to twist 4 operators, and $\varphi_{P}$ and $\varphi_{\sigma}$ to twist 3 ones. Due to the choice of the light-cone gauge $x^{\mu} A_{\mu}(x)=0$, the path-ordered gauge factor $P \exp \left(i g_{s} \int_{0}^{1} d u x^{\mu} A_{\mu}(u x)\right)$ has been omitted. 
Notice that the coefficient in front of the r.h.s. of eqs.(2.6), (2.7) can be written in terms of the light quark condensate $<\bar{u} u>$ using the PCAC relation: $\frac{f_{\pi} m_{\pi}^{2}}{m_{u}+m_{d}}=-\frac{2}{f_{\pi}}<\bar{u} u>$.

The pion matrix elements of quark-gluon operators can also be parameterized in terms of wave functions [16]:

$$
\begin{aligned}
& <\pi(q)\left|\bar{d}(x) \sigma_{\alpha \beta} \gamma_{5} g_{s} G_{\mu \nu}(u x) u(0)\right| 0>= \\
& i f_{3 \pi}\left[\left(q_{\mu} q_{\alpha} g_{\nu \beta}-q_{\nu} q_{\alpha} g_{\mu \beta}\right)-\left(q_{\mu} q_{\beta} g_{\nu \alpha}-q_{\nu} q_{\beta} g_{\mu \alpha}\right)\right] \int \mathcal{D} \alpha_{i} \varphi_{3 \pi}\left(\alpha_{i}\right) e^{i q x\left(\alpha_{1}+v \alpha_{3}\right)} \\
& <\pi(q)\left|\bar{d}(x) \gamma_{\mu} \gamma_{5} g_{s} G_{\alpha \beta}(v x) u(0)\right| 0>= \\
& f_{\pi}\left[q_{\beta}\left(g_{\alpha \mu}-\frac{x_{\alpha} q_{\mu}}{q \cdot x}\right)-q_{\alpha}\left(g_{\beta \mu}-\frac{x_{\beta} q_{\mu}}{q \cdot x}\right)\right] \int \mathcal{D} \alpha_{i} \varphi_{\perp}\left(\alpha_{i}\right) e^{i q x\left(\alpha_{1}+v \alpha_{3}\right)} \\
& +f_{\pi} \frac{q_{\mu}}{q \cdot x}\left(q_{\alpha} x_{\beta}-q_{\beta} x_{\alpha}\right) \int \mathcal{D} \alpha_{i} \varphi_{\|}\left(\alpha_{i}\right) e^{i q x\left(\alpha_{1}+v \alpha_{3}\right)}
\end{aligned}
$$

and

$$
\begin{aligned}
& <\pi(q)\left|\bar{d}(x) \gamma_{\mu} g_{s} \tilde{G}_{\alpha \beta}(v x) u(0)\right| 0>= \\
& i f_{\pi}\left[q_{\beta}\left(g_{\alpha \mu}-\frac{x_{\alpha} q_{\mu}}{q \cdot x}\right)-q_{\alpha}\left(g_{\beta \mu}-\frac{x_{\beta} q_{\mu}}{q \cdot x}\right)\right] \int \mathcal{D} \alpha_{i} \tilde{\varphi}_{\perp}\left(\alpha_{i}\right) e^{i q x\left(\alpha_{1}+v \alpha_{3}\right)} \\
& +i f_{\pi} \frac{q_{\mu}}{q \cdot x}\left(q_{\alpha} x_{\beta}-q_{\beta} x_{\alpha}\right) \int \mathcal{D} \alpha_{i} \tilde{\varphi}_{\|}\left(\alpha_{i}\right) e^{i q x\left(\alpha_{1}+v \alpha_{3}\right)} .
\end{aligned}
$$

The operator $\tilde{G}_{\alpha \beta}$ is the dual of $G_{\alpha \beta}: \tilde{G}_{\alpha \beta}=\frac{1}{2} \epsilon_{\alpha \beta \delta \rho} G^{\delta \rho} ; \mathcal{D} \alpha_{i}$ is defined as $\mathcal{D} \alpha_{i}=$ $d \alpha_{1} d \alpha_{2} d \alpha_{3} \delta\left(1-\alpha_{1}-\alpha_{2}-\alpha_{3}\right)$. The function $\varphi_{3 \pi}$ is twist 3 , while all the wave functions appearing in eqs.(2.9), (2.10) are twist 4 . The wave functions $\varphi\left(x_{i}, \mu\right)$ ( $\mu$ is the renormalization point) describe the distribution in longitudinal momenta inside the pion, the parameters $x_{i}$ $\left(\sum_{i} x_{i}=1\right)$ representing the fractions of the longitudinal momentum carried by the quark and the antiquark.

The wave function normalizations immediately follow from the definitions (2.5)-(2.10): $\int_{0}^{1} d u \varphi_{\pi}(u)=\int_{0}^{1} d u \varphi_{\sigma}(u)=1, \int_{0}^{1} d u g_{1}(u)=\delta^{2} / 12, \int_{0}^{1} d u G_{2}(u)=\delta^{2} / 18\left(\right.$ with $G_{2}$ defined as $\left.G_{2}(u)=-\int_{0}^{u} d v g_{2}(v)\right) \int \mathcal{D} \alpha_{i} \varphi_{\perp}\left(\alpha_{i}\right)=\int \mathcal{D} \alpha_{i} \varphi_{\|}\left(\alpha_{i}\right)=0, \int \mathcal{D} \alpha_{i} \tilde{\varphi}_{\perp}\left(\alpha_{i}\right)=-\int \mathcal{D} \alpha_{i} \tilde{\varphi}_{\|}\left(\alpha_{i}\right)=$ $\delta^{2} / 3$, with the parameter $\delta$ defined by the matrix element: $<\pi(q)\left|\bar{d} g_{s} \tilde{G}_{\alpha \mu} \gamma^{\alpha} u\right| 0>=i \delta^{2} f_{\pi} q_{\mu}$.

In the limit $\mu \rightarrow \infty$ the wave functions assume an asymptotic form which is approximately symmetric in the variables $x_{i}$ and hence represents an almost equal distribution of the 
pion momentum between its constituents. This asymptotic wave function can be calculated by exploiting the conformal invariance of QCD in the short distance region. On the other hand, to investigate the general properties of the wave functions and their deviation from the asymptotic form, non perturbative methods are required. Usually, the momenta of the wave functions are determined by QCD sum rules, and then the wave functions themselves are reconstructed [16,20]. In a calculation of the type presented here, however, the complete form of the pion wave functions is not required, since only the values in particular points, namely near the symmetric point $u_{0}=1 / 2$, are needed. Therefore, the uncertainty in the results is only related to a finite number of input parameters, as it will be shown in the following.

The expansion of the correlator (2.1) near the light-cone produces the following expression for the invariant function $F$ :

$$
\begin{aligned}
F^{Q C D}\left(p^{2},(p+q)^{2}\right) & =f_{\pi} \int_{0}^{1} d u \frac{\varphi_{\pi}(u)}{m_{b}^{2}-(p+u q)^{2}} \\
& -f_{\pi} \int_{0}^{1} d u \frac{4}{\left[m_{b}^{2}-(p+u q)^{2}\right]^{2}}\left[1+\frac{2 m_{b}^{2}}{m_{b}^{2}-(p+u q)^{2}}\right]\left(g_{1}(u)+G_{2}(u)\right) \\
& +2 m_{b} \frac{f_{\pi} m_{\pi}^{2}}{6\left(m_{u}+m_{d}\right)} \int_{0}^{1} d u \frac{\varphi_{\sigma}(u)}{\left[m_{b}^{2}-(p+u q)^{2}\right]^{2}} \\
& +f_{\pi} \int_{0}^{1} d v \int \mathcal{D} \alpha_{i} \frac{\varphi_{\perp}\left(\alpha_{i}\right)-\tilde{\varphi}_{\perp}\left(\alpha_{i}\right)}{\left\{m_{b}^{2}-\left[p+q\left(\alpha_{1}+v \alpha_{3}\right)\right]^{2}\right\}^{2}} \\
& -4 f_{\pi} \int_{0}^{1} d v(v-1) \int_{0}^{1} d \alpha_{3} \hat{\psi}\left(\alpha_{3}\right) \frac{p}{\left\{m_{b}^{2}-\left[p+q\left(1+(v-1) \alpha_{3}\right)\right]^{2}\right\}^{3}} \\
& -4 f_{\pi} \int_{0}^{1} d v \int_{0}^{1} d \alpha_{3} \int_{0}^{1-\alpha_{3}} d \alpha_{1} \hat{\phi}\left(\alpha_{1}, \alpha_{3}\right) \frac{p \cdot q}{\left\{m_{b}^{2}-\left[p+q\left(\alpha_{1}+v \alpha_{3}\right)\right]^{2}\right\}^{3}} \\
& +2 f_{\pi} \int_{0}^{1} v d v \int \mathcal{D} \alpha_{i} \frac{\varphi\left(\alpha_{i}\right)}{\left\{m_{b}^{2}-\left[p+q\left(\alpha_{1}+v \alpha_{3}\right)\right]^{2}\right\}^{2}}
\end{aligned}
$$

where $\hat{\psi}\left(\alpha_{3}\right)=-\int_{0}^{\alpha_{3}} d t \int_{0}^{1-t} \phi\left(\alpha_{1}, t\right) d \alpha_{1}, \hat{\phi}\left(\alpha_{1}, \alpha_{3}\right)=-\int_{0}^{\alpha_{1}} d t \phi\left(t, \alpha_{3}\right)$, and $\phi=\varphi_{\perp}-\tilde{\varphi}_{\perp}+$ $\varphi_{\|}-\tilde{\varphi}_{\|}$. Taking into account the wave function normalizations we recover, in the soft pion limit $q \rightarrow 0$, the expressions obtained in 99 .

The sum rule for $g_{B^{*} B^{*} \pi}$ follows from the approximate equality of the expressions (2.2) and (2.11). Moreover, invoking duality arguments, the contribution of the continuum in (2.2) corresponds to the QCD contribution. This allows to isolate the pole contribution to 
(2.2). The equality between (2.2) and (2.11) can be improved performing a double Borel transform in the variables $-p^{2}$ and $-(p+q)^{2}$. Defining $M_{1}^{2}$ and $M_{2}^{2}$ as the Borel parameters associated to the channels $p$ and $p+q$, respectively, and using the formula:

$$
\mathcal{B}_{M_{1}^{2}} \mathcal{B}_{M_{2}^{2}} \frac{(\ell-1) !}{\left[m_{b}^{2}-(p+u q)^{2}\right]^{\ell}}=\frac{\left(M^{2}\right)^{2-\ell}}{M_{1}^{2} M_{2}^{2}} \exp \left(-\frac{m_{b}^{2}+q^{2}\left(1-u_{0}\right)}{M^{2}}\right) \delta\left(u-u_{0}\right)
$$

with $M^{2}=\frac{M_{1}^{2} M_{2}^{2}}{M_{1}^{2}+M_{2}^{2}}$ and $u_{0}=\frac{M_{1}^{2}}{M_{1}^{2}+M_{2}^{2}}$, we get:

$$
\begin{aligned}
F^{Q C D}\left(M_{1}^{2}, M_{2}^{2}\right) & =\frac{f_{\pi}}{M_{1}^{2} M_{2}^{2}} e^{-\frac{m_{b}^{2}}{M^{2}}}\left\{M^{2} \varphi_{\pi}\left(u_{0}\right)+\frac{m_{b} m_{\pi}^{2}}{3\left(m_{u}+m_{d}\right)} \varphi_{\sigma}\left(u_{0}\right)\right. \\
& -4\left(1+\frac{m_{b}^{2}}{M^{2}}\right)\left[g_{1}\left(u_{0}\right)+G_{2}\left(u_{0}\right)\right] \\
& +\int_{0}^{u_{0}} d \alpha_{1} \int_{u_{0}-\alpha_{1}}^{1-\alpha_{1}} \frac{d \alpha_{3}}{\alpha_{3}}\left(\varphi_{\perp}-\tilde{\varphi}_{\perp}\right)\left(\alpha_{1}, 1-\alpha_{1}-\alpha_{3}, \alpha_{3}\right) \\
& +2 \int_{0}^{u_{0}} d \alpha_{1} \int_{u_{0}-\alpha_{1}}^{1-\alpha_{1}} d \alpha_{3} \frac{u_{0}-\alpha_{1}}{\alpha_{3}^{2}} \varphi_{\|}\left(\alpha_{1}, 1-\alpha_{1}-\alpha_{3}, \alpha_{3}\right) \\
& -\left[\frac{\hat{\psi}\left(1-u_{0}\right)}{1-u_{0}}-\int_{1-u_{0}}^{1} d \alpha_{3} \frac{\hat{\psi}\left(\alpha_{3}\right)}{\alpha_{3}^{2}}\right] \\
& \left.-\left[\int_{0}^{u_{0}} d \alpha_{3} \frac{\hat{\phi}\left(u_{0}-\alpha_{3}, \alpha_{3}\right)}{\alpha_{3}}-\int_{0}^{1} d \alpha_{3} \frac{\hat{\phi}\left(u_{0}, \alpha_{3}\right)}{\alpha_{3}}\right]\right\} .
\end{aligned}
$$

On the other hand the Borel transformed hadronic representation of the function F reads:

$$
F\left(M_{1}^{2}, M_{2}^{2}\right)=\frac{2}{f_{\pi}} \frac{m_{B^{*}}^{2} f_{B^{*}}^{2} g_{B^{*} B^{*} \pi}}{M_{1}^{2} M_{2}^{2}} e^{-m_{B^{*}}^{2}\left(\frac{1}{M_{1}^{2}}+\frac{1}{M_{2}^{2}}\right)}+\int d s d s^{\prime} \rho^{\text {cont }}\left(s, s^{\prime}\right) e^{-\frac{s}{M_{1}^{2}}-\frac{s^{\prime}}{M_{2}^{2}}} .
$$

As shown in (2.14), the Borel transformation exponentially suppresses, for small values of the parameters $M_{1}^{2}, M_{2}^{2}$, the contribution of higher states and of the continuum; moreover, possible subtraction terms in (2.2), which depend only on $p^{2}$ or $(p+q)^{2}$, are removed by the independent borelization in the two channels. This is a feature which renders light-cone sum rules quite appealing, as far as the calculation of the strong couplings is concerned. As a matter of fact, the determination of the strong couplings by, e.g., short-distance rules is usually performed in the soft pion limit $q \rightarrow 0$, thus preventing the possibility of an independent borelization in the two $B^{*}$ channels.

Considering the symmetry of the correlator (2.1) (Fig.1), it is natural to choose $M_{1}^{2}=$ $M_{2}^{2}$, and then $u_{0}=1 / 2$, together with $s_{0}=s_{0}^{\prime}$. Such a choice corresponds to a quark and an 
antiquark of the same momentum inside the pion. In this condition the subtraction of the continuum can be performed, in the leading twist term, with the substitution $e^{-m_{b}^{2} / M^{2}} \rightarrow$ $e^{-m_{b}^{2} / M^{2}}-e^{-s_{0} / M^{2}}$ [16], a recipe we follow considering that the numerical contribution of the higher twist terms is small.

In this way we get the sum rule for $g_{B^{*} B^{*} \pi}$ :

$$
\begin{aligned}
f_{B^{*}}^{2} g_{B^{*} B^{*} \pi} & =\frac{f_{\pi}^{2}}{2 m_{B^{*}}^{2}} e^{\frac{m_{B^{*}}^{2}}{M^{2}}}\left\{\left(e^{-\frac{m_{b}^{2}}{M^{2}}}-e^{-\frac{s_{0}}{M^{2}}}\right) M^{2} \varphi_{\pi}\left(u_{0}\right)\right. \\
& +e^{-\frac{m_{b}^{2}}{M^{2}}}\left[\frac{m_{b} m_{\pi}^{2}}{3\left(m_{u}+m_{d}\right)} \varphi_{\sigma}\left(u_{0}\right)-4\left(1+\frac{m_{b}^{2}}{M^{2}}\right)\left[g_{1}\left(u_{0}\right)+G_{2}\left(u_{0}\right)\right]\right. \\
& +\int_{0}^{u_{0}} d \alpha_{1} \int_{u_{0}-\alpha_{1}}^{1-\alpha_{1}} \frac{d \alpha_{3}}{\alpha_{3}}\left(\varphi_{\perp}-\tilde{\varphi}_{\perp}\right)\left(\alpha_{1}, 1-\alpha_{1}-\alpha_{3}, \alpha_{3}\right) \\
& +2 \int_{0}^{u_{0}} d \alpha_{1} \int_{u_{0}-\alpha_{1}}^{1-\alpha_{1}} d \alpha_{3} \frac{u_{0}-\alpha_{1}}{\alpha_{3}^{2}} \varphi_{\|}\left(\alpha_{1}, 1-\alpha_{1}-\alpha_{3}, \alpha_{3}\right) \\
& -\left[\frac{\hat{\psi}\left(1-u_{0}\right)}{1-u_{0}}-\int_{1-u_{0}}^{1} d \alpha_{3} \frac{\hat{\psi}\left(\alpha_{3}\right)}{\alpha_{3}^{2}}\right] \\
& \left.-\left[\int_{0}^{u_{0}} d \alpha_{3} \frac{\hat{\phi}\left(u_{0}-\alpha_{3}, \alpha_{3}\right)}{\alpha_{3}}-\int_{0}^{1} d \alpha_{3} \frac{\hat{\phi}\left(u_{0}, \alpha_{3}\right)}{\alpha_{3}}\right]\right\}
\end{aligned}
$$

The numerical analysis of this sum rule is described in the next section. Here we want to show that eq.(2.15) has the right behaviour in the limit $m_{b} \rightarrow \infty$ [16], and that an analytical expression can be derived in the asymptotic regime.

In the limit $m_{b} \rightarrow \infty$ the $B^{*}$ meson mass can be related to the $b$ quark mass by the relation $m_{B^{*}}=m_{b}+\Lambda+\mathcal{O}\left(\frac{1}{m_{b}}\right)$, where $\Lambda$ represents the binding energy of the light degrees of freedom in the static $b$ quark chromomagnetic field. Moreover, the $B^{*}$ leptonic constant depends on $m_{b}$ as: $f_{B^{*}}=\frac{\hat{F}}{\sqrt{m_{b}}}$ (modulo logarithmic corrections), with $\hat{F}$ a low energy parameter remaining constant in the infinite $m_{b}$ limit. Rescaling the Borel parameter: $M^{2}=2 m_{b} E$, and the continuum threshold $s_{0}=m_{b}^{2}+2 m_{b} y_{0}$, with $E$ and $y_{0}$ independent of the heavy mass, we recover from (2.15) a sum rule holding in the asymptotic limit:

$$
\hat{F}^{2} g=f_{\pi}^{2} e^{\frac{\Lambda}{E}}\left\{\left(1-e^{-\frac{y_{0}}{E}}\right) E \varphi_{\pi}\left(u_{0}\right)-\frac{<\bar{u} u>}{3 f_{\pi}^{2}} \varphi_{\sigma}\left(u_{0}\right)-\frac{1}{E}\left[g_{1}\left(u_{0}\right)+G_{2}\left(u_{0}\right)\right]\right\},
$$

which is the analogous of the sum rule derived for $g$ [10 by the short distance expansion. Notice that the sum rule $(2.16)$ is not hampered by the problem of the subtraction of the 
so-called "parasitic" contributions, which must be carefully treated in the short-distance calculation [10]; as a matter of fact, it results from two independent borelizations with parameters $E_{1}$ and $E_{2}$, with $u_{0}=\frac{E_{1}}{E_{1}+E_{2}}$ and $E=\frac{E_{1} E_{2}}{E_{1}+E_{2}}$.

Finally, for $m_{b} \rightarrow \infty$, it is worth observing that the light-cone sum rule for $g_{B^{*} B \pi}$ [16] coincides with eq.(2.16), as required by the heavy spin symmetry.

\section{NUMERICAL ANALYSIS FOR $g_{B^{*} B^{*} \pi}$ AND $g$}

The numerical analysis of eqs. (2.15), (2.16) requires a set of light-cone pion wave

functions. Actually, if the choice $u_{0}=\frac{1}{2}$ is made, only the value of the wave functions in the middle point is needed.

Different sets of wave functions are available in the literature. Here we use the functions derived in [20] and adopted in [16] to compute the coupling $g_{B^{*} B \pi}$. At the $b$-quark mass scale the values of the various functions appearing in eq.(2.15), at $u_{0}=\frac{1}{2}$, are: $\varphi_{\pi}\left(u_{0}\right)=1.219$, $\varphi_{\sigma}\left(u_{0}\right)=1.463, g_{1}\left(u_{0}\right)=-3.37 \times 10^{-3} G_{e V} V^{2}$ and $G_{2}\left(u_{0}\right)=-1.8 \times 10^{-2} G e V^{2} ;$ these two last numbers correspond to the choice $\delta^{2}\left(m_{b}\right)=0.17 \mathrm{GeV}^{2}$. The expressions for the functions $\phi$ and $\tilde{\phi}$ can be found in [16]; their contribution to the sum rule corresponds to a small fraction (of the order of $10^{-2}$ ) of the final result.

Using $m_{b}=4.6 \mathrm{GeV}$ [22, $<\bar{u} u>=-(240 \mathrm{MeV})^{3}$ and the threshold $s_{0}$ in the range $s_{0}=33-35 \mathrm{GeV}^{2}$ we get the result depicted in Fig.2. The duality domain in $M^{2}$, where the continuum contribution is small (less than $30 \%$ ), can be chosen as $M^{2}=6-12 \mathrm{GeV}^{2}$. The result is:

$$
f_{B^{*}}^{2} g_{B^{*} B^{*} \pi}=(8.5 \pm 1.5) \times 10^{-3} \mathrm{GeV}^{2},
$$

where the uncertainty is due to the variation with the threshold $s_{0}$ and the Borel parameter $M^{2}$. We do not include the uncertainty on the wave functions, which we consider as external input parameters.

Eq.(2.15) can also be used to compute the coupling $g_{D^{*} D^{*} \pi}$. Using $m_{c}=1.35 \mathrm{GeV}$ 
and $s_{0}=6-7 \mathrm{GeV}^{2}$, taking into account the variation of the wave functions with the renormalization scale, we derive the result in Fig.3. In the range $M^{2}=2-4 \mathrm{GeV}^{2}$ we get:

$$
f_{D^{*}}^{2} g_{D^{*} D^{*} \pi}=(1.8 \pm 0.3) \times 10^{-2} \mathrm{GeV}^{2}
$$

The results (3.1), (3.2) favourably compare with the outcome of short-distance QCD sum rules [9]: $f_{B^{*}}^{2} g_{B^{*} B^{*} \pi}=(9.4 \pm 1.8) \times 10^{-3} \mathrm{GeV}^{2}, f_{D^{*}}^{2} g_{D^{*} D^{*} \pi}=(1.7 \pm 0.4) \times 10^{-2} \mathrm{GeV}^{2}$.

The analogous couplings $B^{*} B \pi, D^{*} D \pi$, computed using the same set of parameters, are:

$$
\begin{aligned}
& f_{B} f_{B^{*}} g_{B^{*} B \pi}=(7.9 \pm 1.0) \times 10^{-3} \mathrm{GeV}^{2} \\
& f_{D} f_{D^{*}} g_{D^{*} D \pi}=(1.5 \pm 0.2) \times 10^{-2} \mathrm{GeV}^{2}
\end{aligned}
$$

to be compared with the short-distance based calculation [10]: $f_{B} f_{B^{*}} g_{B^{*} B \pi}=(7.0 \pm 1.5) \times$ $10^{-3} \mathrm{GeV}^{2}$ and $f_{D} f_{D^{*}} g_{D^{*} D \pi}=(1.2 \pm 0.3) \times 10^{-2} \mathrm{GeV}^{2}$.

The analysis of the asymptotic sum rule (2.16) allows to derive $g$ and, by taking the logarithmic derivative in $1 / E$, the parameter $\Lambda$. Using $y_{0}=1.2-1.4 \mathrm{GeV}$ and $E=$ $0.8-2.0 \mathrm{GeV}$, we get, from the curves depicted in Fig.4:

$$
\begin{aligned}
\Lambda & =0.40 \pm 0.07 \mathrm{GeV} \\
\hat{F}^{2} g & =0.031 \pm 0.006 \mathrm{GeV}^{3}
\end{aligned}
$$

the analogous result obtained in [10] is $\hat{F}^{2} g=0.035 \pm 0.008 \mathrm{GeV}^{3}$.

The calculation of the couplings can be done, using eqs. 3.1 3.4), once the leptonic constants are known. Since the correlator (2.1) has been computed at the leading order in $\alpha_{s}$, it is consistent to use the leptonic constants determined at the same order. Then, one can use [23]: $f_{B^{*}}=190 \pm 10 \mathrm{MeV}, f_{D^{*}}=220 \pm 24 \mathrm{MeV}, f_{B}=150 \pm 20 \mathrm{MeV}, f_{D}=170 \pm 10 \mathrm{MeV}$, with the results:

$$
\begin{aligned}
g_{B^{*} B^{*} \pi} & =0.24 \pm 0.05, & g_{D^{*} D^{*} \pi} & =0.31 \pm 0.08 \\
g_{B^{*} B \pi} & =0.28 \pm 0.05 & & g_{D^{*} D \pi}=0.40 \pm 0.07
\end{aligned}
$$

To derive the value of $g$, in the limit $m_{b} \rightarrow \infty$, one can linearly extrapolate the above results: 


$$
\begin{aligned}
g_{P^{*} P^{*} \pi} & =g\left(1+\frac{a}{m_{Q}}\right) \\
g_{P^{*} P \pi} & =g\left(1+\frac{b}{m_{Q}}\right),
\end{aligned}
$$

obtaining:

$$
\begin{array}{ll}
g=0.21 \pm 0.07 \quad, \quad a=0.6 \pm 0.9 \mathrm{GeV} \\
g=0.23 \pm 0.08 \quad, \quad b=1.0 \pm 1.0 \mathrm{GeV}
\end{array}
$$

from $g_{P^{*} P^{*} \pi}$ and $g_{P^{*} P \pi}$, respectively. The results (3.11), (3.12) show that the same value for $g$ is obtained by extrapolating to $m_{b} \rightarrow \infty$ the values obtained for $g_{P^{*} P^{*} \pi}$ and $g_{P^{*} P \pi}$. One can conservatively quote $g=0.22 \pm 0.10$ as the result of the extrapolation. It is also worth observing that, although the fit does not accurately fix the parameters $a, b$, the $1 / m_{Q}$ corrections are rather sizable in the case of the charm quark.

Let us now consider the calculation of $g$ from eq.(3.6). Using the determination in [24]: $\hat{F}=0.30 \pm 0.05$, obtained neglecting radiative $\mathcal{O}\left(\alpha_{s}\right)$ corrections, one gets:

$$
g=0.34 \pm 0.10
$$

a result which, although higher in the central value, is compatible with the outcome of the fit.

It is interesting to consider the inclusion of $O\left(\alpha_{s}\right)$ corrections in the leptonic constants, to investigate at least partially the role of the perturbative corrections in the determination of $g$. Using, therefore, $f_{B^{*}}=213 \pm 34 \mathrm{MeV}, f_{D^{*}}=258 \pm 26 \mathrm{MeV}, f_{B}=180 \pm 30 \mathrm{MeV}$, $f_{D}=195 \pm 20 \mathrm{MeV}$ 23], one gets:

$$
\begin{array}{ll}
g_{B^{*} B^{*} \pi}=0.19 \pm 0.04, & g_{D^{*} D^{*} \pi}=0.23 \pm 0.05 \\
g_{B^{*} B \pi}=0.21 \pm 0.06, & g_{D^{*} D \pi}=0.30 \pm 0.06
\end{array}
$$

i.e.:

$$
\begin{aligned}
& g=0.17 \pm 0.06 \quad, \quad a=0.6 \pm 0.8 \mathrm{GeV} \\
& g=0.17 \pm 0.08 \quad, \quad b=1.0 \pm 1.4 \mathrm{GeV}
\end{aligned}
$$


The inclusion of the $\mathcal{O}\left(\alpha_{s}\right)$ terms in the leptonic constants does not spoil the prediction that the same $g$ is obtained from the $B^{*} B^{*} \pi$ and $B^{*} B \pi$ channels. The numerical value of $g$ results modified at the level of $30 \%$.

Several determinations of the coupling $g$ can be found in the literature [10,9, 15, 16, 25, 26], with values varying up to $g=1$. The analysis reported here points towards small values of $g$, in agreement with the outcome of the relativistic potential model [25], which shows that the nonrelativistic result $g=1$ is reduced $(g \simeq 1 / 3)$ if a relativistic treatment of the light quarks is adopted.

\section{LIGHT-CONE SUM RULES FOR $g_{B_{1} B_{0} \pi}$ AND $g^{\prime}$}

The same method described in the previous sections can be used to determine the cou-

pling $g^{\prime}$, which weightes in the lagrangian (1.1) the strong interaction between positive parity heavy mesons and pions. For a finite mass of the heavy quark, the coupling $g_{B_{1} B_{0} \pi}$ can be obtained from the correlator of a scalar $J_{S}=\bar{q} Q$ and an axial $J_{a}^{\mu}=\bar{q} \gamma^{\mu} \gamma_{5} Q$ current:

$$
\begin{aligned}
G_{\mu}(p, p+q) & =i \int d x<\pi^{-}(q)\left|T\left[\bar{d}(x) \gamma_{\mu} \gamma_{5} b(x), \bar{b}(0) u(0)\right]\right| 0>e^{i p x} \\
& =G^{(0)}(p+q)_{\mu}+G^{(1)} q_{\mu}
\end{aligned}
$$

since the invariant function $G^{(1)}$ gets contribution from the poles $B_{0}$ and $B_{1}$. Defining

$$
<\pi(q) B_{0}(p) \mid B_{1}(p+q, \epsilon)>=\frac{2 \sqrt{m_{B_{0}} m_{B_{1}}}}{f_{\pi}} g_{B_{1} B_{0} \pi} \epsilon \cdot q
$$

with $m_{B_{1}}$ and $m_{B_{0}}$ the masses of the positive $1^{+}$and $0^{+}$states, respectively, and

$$
\begin{gathered}
<0|\bar{b} u| B_{0}>=\frac{f_{B_{0}} m_{B_{0}}^{2}}{m_{b}}, \\
<0\left|\bar{d} \gamma_{\mu} \gamma_{5} b\right| B_{1}(p, \epsilon)>=f_{B_{1}} m_{B_{1}} \epsilon_{\mu},
\end{gathered}
$$

we get the Borel transformed sum rule for $g_{B_{1} B_{0} \pi}$ : 


$$
\begin{aligned}
g_{B_{1} B_{0} \pi} & \frac{2 f_{B_{1}} m_{B_{1}}^{\frac{3}{2}} f_{B_{0}} m_{B_{0}}^{\frac{5}{2}}}{f_{\pi} m_{b}}=e^{\frac{m_{B_{0}}^{2}+m_{B_{1}}^{2}}{2 M^{2}}}\left\{\left(e^{-m_{b}^{2} / M^{2}}-e^{-s_{0}^{2} / M^{2}}\right) M^{2} m_{b} f_{\pi} \varphi_{\pi}\left(u_{0}\right)\right. \\
+ & e^{-m_{b}^{2} / M^{2}}\left[M^{2}\left[\frac{f_{\pi} m_{\pi}^{2}}{m_{u}+m_{d}}\left(1-u_{0}\right) \varphi_{P}\left(u_{0}\right)-\frac{f_{\pi} m_{\pi}^{2}}{6\left(m_{u}+m_{d}\right)}\left(2 \varphi_{\sigma}\left(u_{0}\right)-\left(1-u_{0}\right) \frac{d \varphi_{\sigma}}{d u}\left(u_{0}\right)\right)\right]\right. \\
- & {\left[2 m_{b} f_{\pi}\left(1-u_{0}\right) g_{2}\left(u_{0}\right)+\frac{f_{\pi} m_{\pi}^{2}}{6\left(m_{u}+m_{d}\right)} 2 m_{b}^{2} \varphi_{\sigma}\left(u_{0}\right)\right] } \\
- & \frac{1}{M^{2}} 4 m_{b}^{3} f_{\pi}\left[g_{1}\left(u_{0}\right)+G_{2}\left(u_{0}\right)\right] \\
- & m_{b} f_{\pi} \int_{0}^{u_{0}} d \alpha_{1} \int_{u_{0}-\alpha_{1}}^{1-\alpha_{1}} d \alpha_{3}\left(\varphi_{\|}+\tilde{\varphi}_{\|}-2 \varphi_{\perp}-2 \tilde{\varphi}_{\perp}\right)\left(\alpha_{1}, 1-\alpha_{1}-\alpha_{3}, \alpha_{3}\right) \\
- & 2 M^{2} f_{3 \pi}\left[\int_{0}^{u_{0}} \frac{d \alpha_{3}}{\alpha_{3}} \varphi_{3 \pi}\left(u_{0}-\alpha_{3}, 1-u_{0}, \alpha_{3}\right)\right. \\
- & \left.\left.\left.\int_{0}^{u_{0}} d \alpha_{1} \int_{u_{0}-\alpha_{1}}^{1-\alpha_{1}} \frac{d \alpha_{3}}{\alpha_{3}^{2}} \varphi_{3 \pi}\left(\alpha_{1}, 1-\alpha_{1}-\alpha_{3}, \alpha_{3}\right)\right]\right]\right\} .
\end{aligned}
$$

In the infinite heavy quark mass limit, it is easy to derive from (4.5) the sum rule for $g^{\prime}$, using the scaling law $f_{B_{1}}=\frac{\hat{F}^{+}}{\sqrt{m_{b}}}$ :

$$
\hat{F}^{+2} g^{\prime}=\frac{f_{\pi}}{2} e^{\frac{\Lambda^{\prime}}{E}}\left\{\left(1-e^{-\frac{y_{0}}{E}}\right) 2 E f_{\pi} \varphi_{\pi}\left(u_{0}\right)+\frac{2}{3} \frac{\bar{u} u>}{f_{\pi}} \varphi_{\sigma}\left(u_{0}\right)-\frac{2 f_{\pi}}{E}\left[g_{1}\left(u_{0}\right)+G_{2}\left(u_{0}\right)\right]\right\} .
$$

$E$ is the Borel parameter; $\Lambda^{\prime}$ is the binding energy for the $s_{\ell}=\frac{1}{2}^{+}$doublet: $\Lambda^{\prime}=m_{B_{1,0}}-m_{b}$, and it can be obtained by the logarithmic derivative of (4.6).

The numerical analysis of the sum rule (4.5) can be carried out using the same set of parameters as in Sect. III. Using $m_{B_{1}}=m_{B_{0}}=5.7 \mathrm{GeV}$ and $s_{0}=36-38 \mathrm{GeV}^{2}$ in the case of beauty, and $m_{D_{1}}=m_{D_{0}}=2.4 \mathrm{GeV}$ and $s_{0}=7-9 \mathrm{GeV}^{2}$ for the charmed mesons, we get:

$$
\begin{aligned}
& f_{B_{1}} f_{B_{0}} g_{B_{1} B_{0} \pi}=(3.4 \pm 0.3) \times 10^{-3} \mathrm{GeV}^{2} \\
& f_{D_{1}} f_{D_{0}} g_{D_{1} D_{0} \pi}=(3.8 \pm 0.5) \times 10^{-3} \mathrm{GeV}^{2}
\end{aligned}
$$

The above equations allow to determine $g_{P_{1} P_{0} \pi}$, since [23]: $f_{B_{1}}=180 \pm 30 \mathrm{MeV}, f_{B_{0}}=$ $180 \pm 30 \mathrm{MeV}, f_{D_{1}}=240 \pm 20 \mathrm{MeV}, f_{D_{0}}=170 \pm 20 \mathrm{MeV}:$

$$
g_{B_{1} B_{0} \pi}=0.11 \pm 0.03 \quad g_{D_{1} D_{0} \pi}=0.09 \pm 0.02
$$


In the infinite heavy quark limit, the result of a linear extrapolation, $g_{P_{1} P_{0} \pi}=g^{\prime}\left(1+\frac{c}{m_{Q}}\right)$, is:

$$
g^{\prime}=0.11 \pm 0.04 \quad c=-0.2 \pm 0.5 \mathrm{GeV} .
$$

The result of the asymptotic sum rule (4.6) is depicted in Fig.5. Varying the threshold parameter $y_{0}$ in the range $y_{0}=1.3-1.5 \mathrm{GeV}$, and the Borel parameter $E$ in the range $E=1.0-2.0 \mathrm{GeV}$, we get:

$$
\begin{aligned}
\Lambda^{\prime} & =0.89 \pm 0.05 \mathrm{GeV} \\
\hat{F}^{+2} g^{\prime} & =0.022 \pm 0.003 \mathrm{GeV}^{3} .
\end{aligned}
$$

With $\hat{F}^{+}=0.46 \pm 0.06 \mathrm{GeV}^{\frac{3}{2}}$ [23], the last equation corresponds to:

$$
g^{\prime}=0.10 \pm 0.02
$$

\section{LIGHT-CONE QCD SUM RULE FOR $g_{B_{1} B^{*} \pi}$ AND $h$}

To complete our analysis of the couplings appearing in (1.1) we consider the parameter $h$ corresponding to the couplings $B_{1} B^{*} \pi$ and $B_{0} B \pi$. The coupling $g_{B_{0} B \pi}$ has been computed by light-cone and short-distance QCD sum rules in [11], while a determination of $g_{B_{1} B^{*} \pi}$ can be found in [17]. Here we want to derive an expression for $h$.

Let us define $g_{B_{1} B^{*} \pi}$ by the matrix element

$$
<B^{*}(p, \epsilon) \pi(q) \mid B_{1}(p+q, \eta)>=g_{B_{1} B^{*} \pi}\left(\epsilon^{*} \cdot \eta\right) \frac{p \cdot q}{m_{B_{1}}}+(p \cdot \eta)\left(q \cdot \epsilon^{*}\right) F
$$

$g_{B_{1} B^{*} \pi}$ is related to the coupling $h$ in eq.(1.1) by:

$$
g_{B_{1} B^{*} \pi}=-\frac{2 h}{f_{\pi}} \sqrt{m_{B_{1}} m_{B^{*}}},
$$

whereas the structure $F$ is subleading in the $m_{Q} \rightarrow \infty$ limit. To calculate $g_{B_{1} B^{*} \pi}$ one has to consider the correlator of an axial and a vector current: 


$$
\begin{aligned}
H_{\mu \nu}(p, p+q) & =i \int d x<\pi^{-}(q)\left|T\left[\bar{d}(x) \gamma_{\mu} \gamma_{5} b(x), \bar{b}(0) \gamma_{\nu} u(0)\right]\right| 0>e^{i p x} \\
& =H^{(0)} g_{\mu \nu}+H^{(1)} p_{\mu} p_{\nu}+\ldots
\end{aligned}
$$

The $1^{+}$and $1^{-}$poles contribute to the invariant function $H^{(0)}$, which must be considered to derive $g_{B_{1} B^{*} \pi}$. One obtains the Borel transformed sum rule:

$$
\begin{aligned}
g_{B_{1} B^{*} \pi} & \left(m_{B_{1}}-m_{B^{*}}\right) f_{B_{1}} m_{B_{1}} f_{B^{*}} m_{B^{*}} e^{-\frac{m_{B^{*}+m_{B_{1}}^{2}}^{2}}{2 M^{2}}}= \\
= & f_{\pi}\left\{\left(e^{-\frac{m_{b}^{2}}{M^{2}}}-e^{-\frac{s_{0}}{M^{2}}}\right)\left(-\frac{M^{4}}{2} \varphi_{\pi}^{\prime}\left(u_{0}\right)+\frac{m_{b} m_{\pi}^{2}}{m_{u}+m_{d}} M^{2} \varphi_{P}\left(u_{0}\right)\right)+e^{-m_{b}^{2} / M^{2}}\left[2 m_{b}^{2} g_{2}\left(u_{0}\right)\right.\right. \\
+ & 2 M^{2}\left(1+\frac{m_{b}^{2}}{M^{2}}\right)\left[G_{2}^{\prime}\left(u_{0}\right)+g_{1}^{\prime}\left(u_{0}\right)\right] \\
+ & M^{2}\left[\int_{0}^{u_{0}} \frac{d \alpha_{3}}{2 \alpha_{3}}\left(\varphi_{\|}+\tilde{\varphi}_{\|}\right)\left(u_{0}-\alpha_{3}, 1-u_{0}, \alpha_{3}\right)+\int_{0}^{1} \frac{d \alpha_{3}}{2 \alpha_{3}}\left(\varphi_{\|}-\tilde{\varphi}_{\|}\right)\left(u_{0}, 1-u_{0}-\alpha_{3}, \alpha_{3}\right)\right. \\
- & \left.\left.\left.\int_{0}^{u_{0}} d \alpha_{1} \int_{u_{0}-\alpha_{1}}^{1-\alpha_{1}} \frac{d \alpha_{3}}{\alpha_{3}^{2}} \varphi_{\|}\left(\alpha_{1}, 1-\alpha_{1}-\alpha_{3}, \alpha_{3}\right)\right]\right]\right\}
\end{aligned}
$$

Differently from the calculation of $g, g^{\prime}$, the determination of $h$ does not concern a symmetric correlator (Fig.1). However, also in this case the choice $u_{0}=1 / 2$ allows us to use the same values of the pion wave functions employed for $g, g^{\prime}$, which reduces the uncertainty related to the wave functions. Considering that $\varphi_{\pi}^{\prime}\left(\frac{1}{2}\right)=g_{2}\left(\frac{1}{2}\right)=G_{2}^{\prime}\left(\frac{1}{2}\right)=g_{1}^{\prime}\left(\frac{1}{2}\right)=0$, in the limit $m_{b} \rightarrow \infty$ one obtains from eq.(5.4) the expression:

$$
\hat{F}^{+} \hat{F} h=-\frac{f_{\pi}^{2} m_{\pi}^{2}}{m_{u}+m_{d}} \frac{E}{\Lambda^{\prime}-\Lambda}\left(1-e^{-y_{0} / E}\right) e^{\frac{\Lambda^{\prime}+\Lambda}{2 E}} \varphi_{P}\left(u_{0}\right)
$$

Eq.(5.5) coincides with the expression that can be obtained from the sum rule for $g_{B_{0} B \pi}$ [11].

The numerical results from eq. (5.4), using the same set of parameters chosen in Sections III, IV:

$$
f_{B_{1}} f_{B^{*}} g_{B_{1} B^{*} \pi}=1.94 \pm 0.37 \mathrm{GeV}^{2}, \quad f_{D_{1}} f_{D^{*}} g_{D_{1} D^{*} \pi}=1.12 \pm 0.19 \mathrm{GeV}^{2}
$$

produce the following values for $g_{P_{1} P^{*} \pi}$ :

$$
g_{B_{1} B^{*} \pi}=56.7 \pm 14.7, \quad g_{D_{1} D^{*} \pi}=21.2 \pm 2.9
$$

The linear extrapolation to $m_{b} \rightarrow \infty: h_{P_{1} P^{*} \pi}=h\left(1+\frac{d}{m_{Q}}\right)$ determines the coupling $h$ : 


$$
h=-0.7 \pm 0.3 \quad, \quad d=-0.2 \pm 0.5 \mathrm{GeV} .
$$

The same result comes from eq.(5.5); as a matter of fact, choosing $y_{0}=1.3-1.5 \mathrm{GeV}$ and the mass parameters $\Lambda, \Lambda^{\prime}$ derived in the previous sections, the result (Fig.6):

$$
\hat{F} \hat{F}^{+} h=-0.083 \pm 0.005 G e V^{3}
$$

corresponds to

$$
h=-0.60 \pm 0.13 \text {, }
$$

in agreement with the determination $h=-0.56 \pm 0.28$ obtained in [11] from $g_{B_{0} B \pi}$.

\section{CONCLUSIONS}

The strong couplings of heavy mesons to pions are expected to obey symmetry relations in the $m_{Q} \rightarrow \infty$ limit, when QCD displays invariance under rotations in the heavy quark spin and flavour space. In this limit, it can be shown that the number of independent couplings reduces to a set of effective parameters.

We employed light-cone sum rules to evaluate the couplings $g_{P^{*} P^{*} \pi}, g_{P^{*} P_{1} \pi}, g_{P_{1} P_{0} \pi}$, $(P=D, B)$ both for finite values of the heavy quark mass and in the infinite mass limit. This procedure allowed us to observe that the light-cone sum rule method reproduces the right $m_{Q}$ scaling behaviour for the physical quantities. Moreover, we verified that the asymptotic results agree with usual QCD sum rules based on the short distance expansion. This

comparison enforces our confidence in the reliability of both the variants of the QCD sum rule tecnique (e.g. short distance based and light cone sum rules), which are among the few available instruments to investigate non perturbative aspects of QCD.

\section{Acknowledgments}

We thank G.Nardulli and N.Paver for interesting discussions. 


\section{REFERENCES}

[1] M.B.Voloshin and M.A.Shifman, Sov. J. Nucl. Phys. 45 (1987) 292; 47 (1988) 511. H.Politzer and M.B.Wise, Phys. Lett. B 206 (1988) 681; B 202 (1988) 504. N.Isgur and M.B.Wise, Phys. Lett. B 232 (1989) 113; B 237 (1990) 527. H.Georgi, Phys. Lett. B 240 (1990) 447. B.Grinstein, Nucl. Phys. B 339 (1990) 253. E.Eichten and B.Hill, Phys. Lett. B 234 (1990) 511. J.D.Bjorken, in "Results and Perspectives in Particle Physics", Proceedings of the 4th Rencontres de Physique, La Thuile, Italy, 1990, edited by M.Greco, Ed. Frontieres, France (1990) page 583.

[2] For a review see: H.Georgi, Proceedings of the TASI Workshop, Boulder, June 2-28, 1991, edited by R.K.Ellis, C.T.Hill and J.D.Likken, World Scientific (1992) page 589. B.Grinstein, Proceedings of the TASI Workshop, Boulder, 29 May - 24 June 1994, edited by J.Donoghue, World Scientific (1992) page 307. M.Neubert, Phys. Rep. 245 (1994) 259.

[3] For a review see: H.Leutwyler, in Chiral Dynamics Workshop (1995), edited by A.M. Bernstein and B.R. Holstein, Springer-Verlag, page 14.

[4] M.B.Wise, Phys. Rev. D 45 (1992) R2188. G.Burdman and J.F.Donoghue, Phys. Lett. B 280 (1992) 287. P.Cho, Phys. Lett. B 285 (1992) 145. H.-Y.Cheng, C.-Y.Cheung, G.L.Lin, Y.C.Lin and H.-L.Yu, Phys. Rev. D 46 (1992) 1148. R.Casalbuoni, A.Deandrea, N.Di Bartolomeo, F.Feruglio, R.Gatto and G.Nardulli, Phys. Lett. B 299 (1993) 139.

[5] N.Isgur and M.B.Wise, Phys. Rev. Lett. 66 (1991) 1130; Phys. Rev. D 43 (1991) 819. U.Kilian, J.G.Körner and D.Pirjol, Phys. Lett. B 288 (1992) 360. A.F.Falk and M.Luke, Phys. Lett. B 292 (1992) 119.

[6] For a review see: R.Casalbuoni, A.Deandrea, N.Di Bartolomeo, F.Feruglio, R.Gatto and G.Nardulli, Phys. Rep. 281 (1997) 145.

[7] C.G.Boyd and B.Grinstein, Nucl. Phys. B 442 (1995) 205. 
[8] J.L. Rosner and M.B. Wise, Phys. Rev. D 47 (1993) 343. L. Randall and E. Sather, Phys. Lett. B 303 (1993) 345. E. Jenkins, Nucl. Phys. B 412 (1994) 181. H.-Y. Cheng, C.-Y. Cheung, G.-L. Lin, Y.C. Lin, T.M. Yan and H.-L. Yu, Phys. Rev. D 49 (1994) 181.

[9] N. Di Bartolomeo, F. Feruglio, R. Gatto and G. Nardulli, Phys. Lett. B 347 (1995) 405.

[10] P. Colangelo, G.Nardulli, A.Deandrea, N.Di Bartolomeo, R.Gatto and F.Feruglio, Phys. Lett. B 339 (1994) 151.

[11] P. Colangelo, F. De Fazio, N. Di Bartolomeo, R. Gatto and G. Nardulli, Phys. Rev. D $52(1995) 6422$.

[12] Y.B.Dai et al., report BIHEP-TH-97-005 (hep-ph/9705223).

[13] M.A. Shifman, A.I. Vainshtein and V.I. Zakharov, Nucl. Phys. B 174 (1979) 385, 448, 519. For a review see: Vacuum structure and QCD sum rules, edited by M. Shifman (North Holland, Amsterdam, 1992).

[14] V.L.Eletski and Y.I.Kogan, Z.Phys. C 28 (1985) 155. A.G.Grozin and O.I.Yakovlev, preprint BUDKERINP-94-3 (February 1994) (hep-ph/9401267).

[15] S.Narison and H.G.Dosch, Phys. Lett. B 368 (1996) 163.

[16] V.M.Belyaev, V.M.Braun, A.Khodjamirian and R.Rückl, Phys. Rev. D 51 (1995) 6177.

[17] T.M. Aliev, N.K. Pak and M.Savci, Phys. Lett. B 390 (1997) 335.

[18] V.L.Chernyak and A.R.Zhitnitsky, JETP Lett. 25, 510 (1977); Yad. Fiz. 31, 1053 (1980). A.V.Efremov and A.V.Radyushkin, Phys. Lett. B 94, 245 (1980); Teor. Mat. Fiz. 42, 147 (1980). G.P.Lepage and S.J.Brodsky, Phys. Lett. B 87, 359 (1979); Phys. Rev. D 22, 2157 (1980).

[19] N.S.Craigie and J.Stern, Nucl. Phys. B 305 (1983) 109. V.L.Chernyak and 
A.R.Zhitnitsky, Phys. Rep. 112, 173 (1984). I.I.Balitsky, V.M.Braun and A.V.Kolesnichenko, Nucl. Phys. B 312 (1989) 509. V.M.Braun and I.E.Filyanov, Z. Phys. C 44 (1989) 157. V.L.Chernyak and A.R.Zhitnitsky, Nucl. Phys. B 345 (1990) 137.

[20] V.M.Braun and I.E.Filyanov, Z. Phys. C 48 (1990) 239.

[21] A.Khodjamirian and R.Rückl, Nucl. Instrum. Meth. A 368 (1995) 28. V.M.Braun and P.Ball, Phys. Rev. D 55 (1997) 5561.

[22] A.Pich and M.Jamin, report IFIC/97-06, FTUV/97-06, HD-THEP-96-55 hepph/9702276).

[23] P.Colangelo, G.Nardulli, A.A.Ovchinnikov and N.Paver, Phys. Lett. B 269 (1991) 204. P.Colangelo, G.Nardulli and N.Paver, Phys. Lett. B 293 (1992) 207.

[24] M.Neubert, Phys. Rev. D 46 (1992) 1076.

[25] P. Colangelo, F. De Fazio and G. Nardulli, Phys. Lett. B 334 (1994) 175.

[26] S. Nussinov and W. Wetzel, Phys. Rev. D 36 (1987) 130. T.-M. Yan et al., Phys. Rev. D 46 (1992) 1148. P. Cho and H. Georgi, Phys. Lett. B 296 (1992) 408. J.F. Amundson et al., Phys. Lett. B 296 (1992) 415. P. Colangelo, F. De Fazio and G. Nardulli, Phys. Lett. B 316 (1993) 555. W.A. Bardeen and C.T. Hill, Phys. Rev. D 49 (1994) 409. P.J. O’Donnel and Q.P. Xu, Phys. Lett. B 336 (1994) 113. H.-Y. Cheng, Phys. Lett. B 399 (1997) 281 . 


\section{FIGURE CAPTIONS}

\section{Fig. 1}

The correlator in eq.(2.1).

\section{Fig. 2}

Stability curve for $f_{B^{*}}^{2} g_{B^{*} B^{*} \pi}$. The curves correspond to the threshold $s_{0}=34 \mathrm{GeV}^{2}$ (continuous line), $s_{0}=35 \mathrm{GeV}^{2}$ (dashed line), $s_{0}=36 \mathrm{GeV}^{2}$ (dotted line).

\section{Fig. 3}

Stability curve for $f_{D^{*}}^{2} g_{D^{*} D^{*} \pi}$. The curves correspond to $s_{0}=6 \mathrm{GeV}^{2}$ (continuous line), $s_{0}=6.5 \mathrm{GeV}^{2}$ (dashed line), $s_{0}=7 \mathrm{GeV}^{2}$ (dotted line).

\section{Fig. 4}

Stability curve for $\Lambda$ and for $\hat{F}^{2} g$. The curves correspond to the threshold $y_{0}=1.2 \mathrm{GeV}$ (continuous line), $y_{0}=1.3 \mathrm{GeV}$ (dashed line), $y_{0}=1.4 \mathrm{GeV}$ (dotted line).

\section{Fig. 5}

Stability curve for $\Lambda^{\prime}$ and for $\hat{F}^{+2} g^{\prime}$. The curves correspond to $y_{0}=1.3 \mathrm{GeV}$ (continuous line), $y_{0}=1.4 \mathrm{GeV}$ (dashed line), $y_{0}=1.5 \mathrm{GeV}$ (dotted line).

\section{Fig. 6}

Stability curve for $\hat{F} \hat{F}^{+} h$. The curves correspond to $y_{0}=1.3 \mathrm{GeV}$ (continuous line), $y_{0}=$ $1.4 \mathrm{GeV}$ (dashed line), $y_{0}=1.5 \mathrm{GeV}$ (dotted line). 


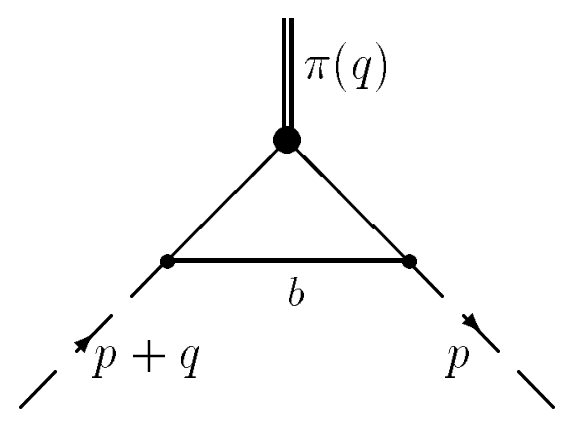

Fig. 1 


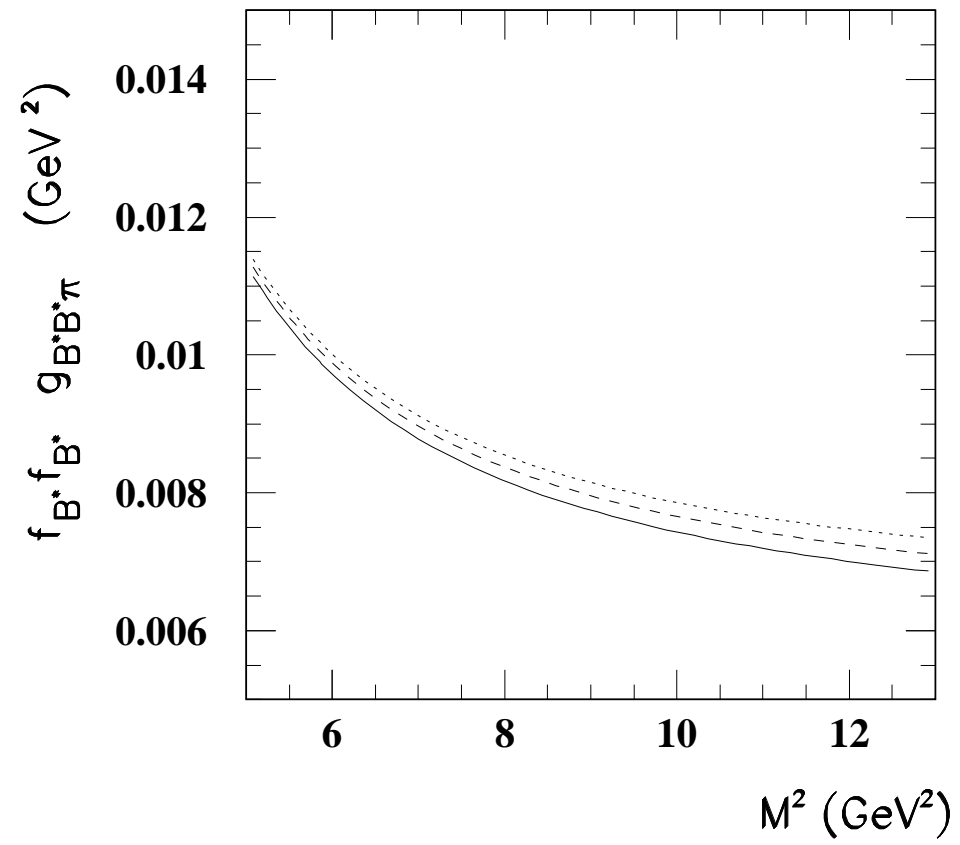

Fig.2 


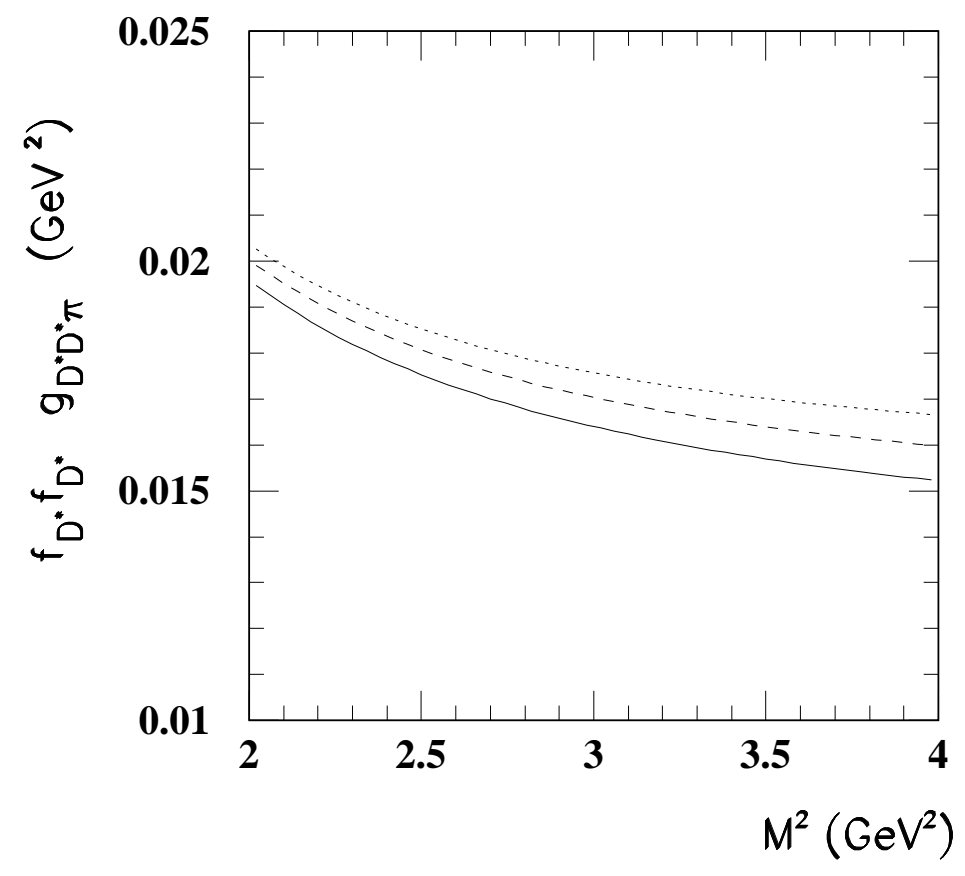

Fig. 3 

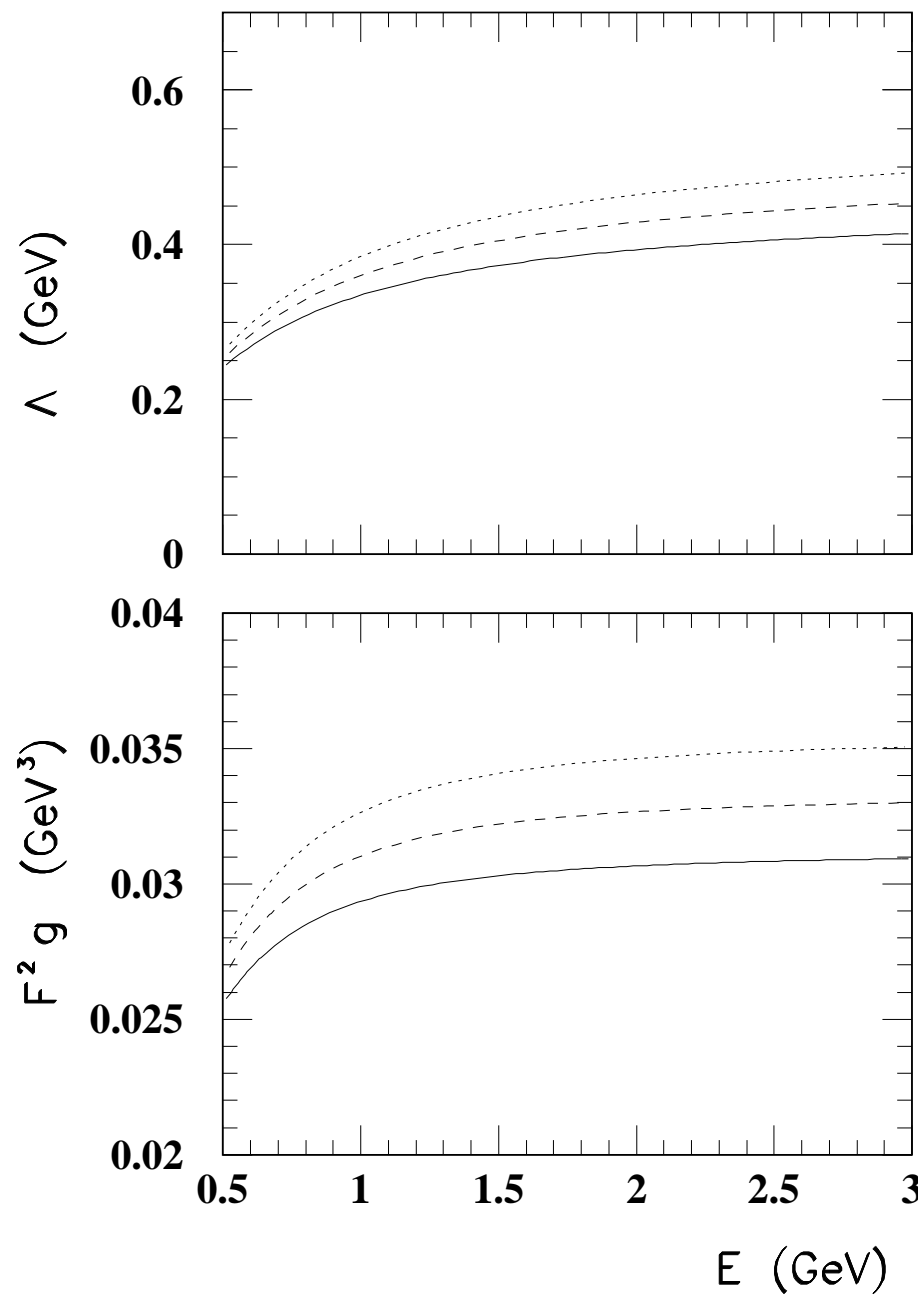

Fig. 4 

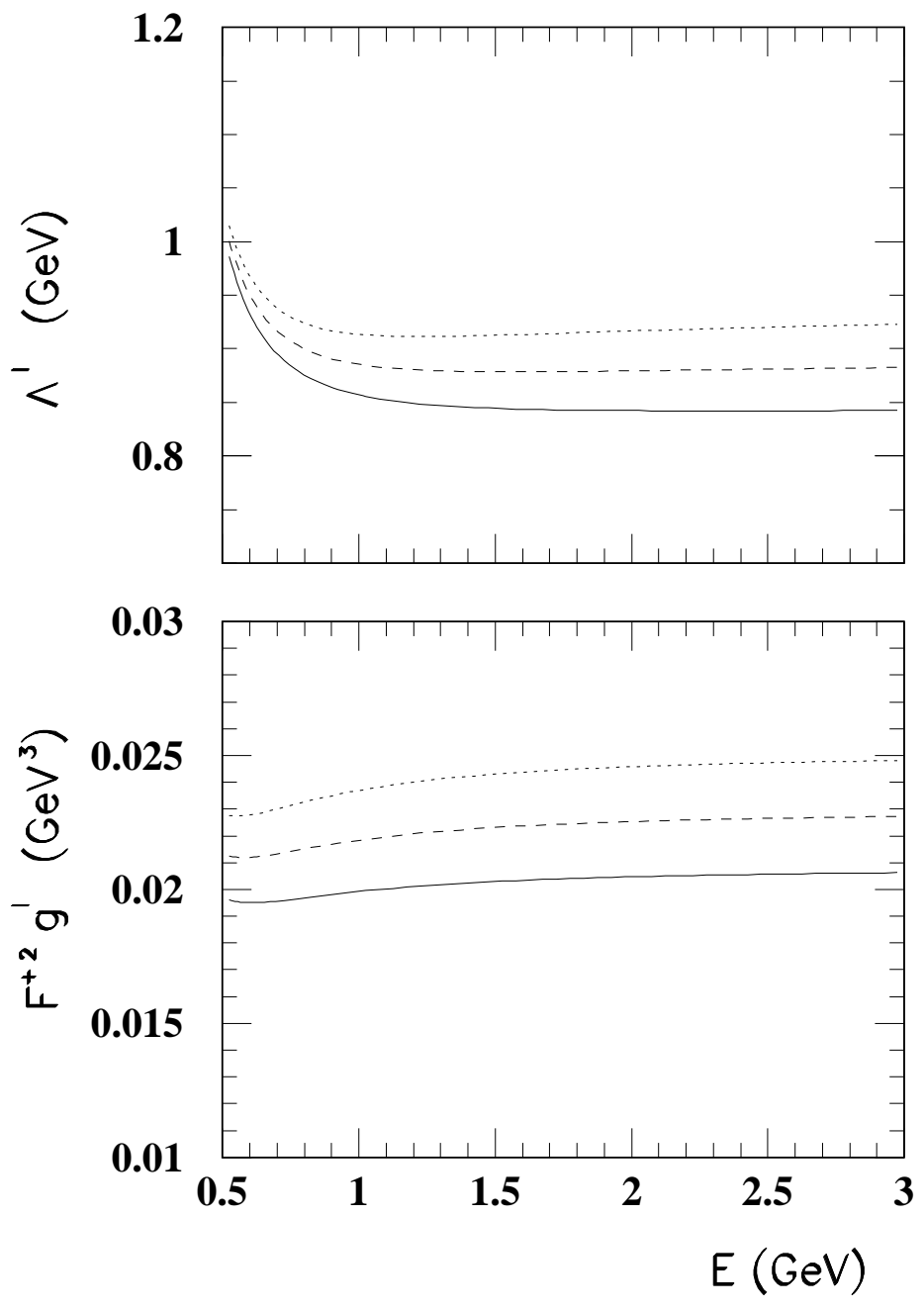

Fig. 5 


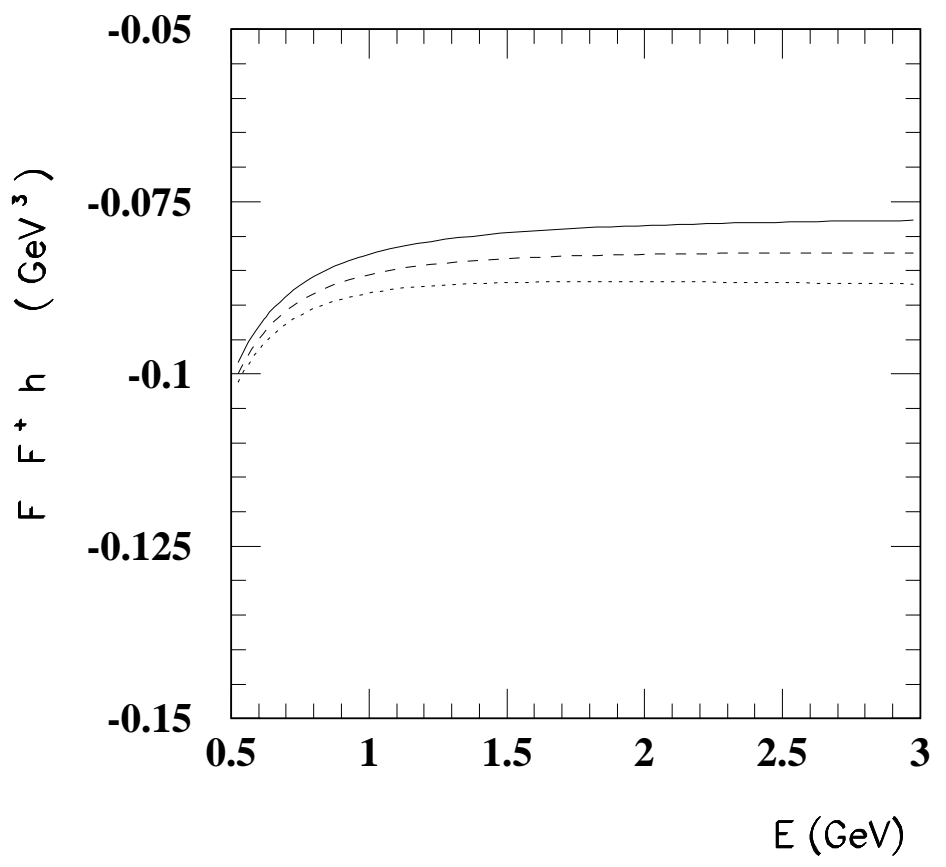

Fig. 6 